\title{
Disrupting the Digital: An Architecture of Parts
}

\author{
MOLLIE CLAYPOOL \\ University College London
}

The paper ascribes to a belief that architecture should be wholly digital - from the scale of the micron and particle to the brick, beam and building, from design to fabrication or construction. This embodies a fundamental and disruptive shift in architecture and design thinking that is unique to the project images included, enabling design to become more inclusive, participatory and open-source. Architecture that is wholly digital requires a radical rethinking of existing design and building practices. Thes projects described in this paper each develops a set of parts in relationship to a specific digital fabrication technology. These parts are defined as open-ended, universal and versatile building blocks, with a digital logic of connectivity. Each physical part has a malefemale connection which is the equivalent of the 0 and 1 in digital data. The design possibilities - or the way that parts can combine and aggregate - can be defined by the geometry and therefore, design agency, of the piece itself. This discrete method advances a theoretical argument about the nature of digital design as needing to be fundamentally discrete, and at the same time responding to ideas coming from open-source, distributed modes methods of production. Furthermore it responds to today's housing crisis, providing for a more democratic and equitable framework for the production of housing. To think of architecture as wholly digital is to substantially disrupt the way that we think about design, authorship, ownership and process, as well as the building technologies and practices we use in contemporary architectural production.

INTRODUCTION - WE SHOULD BE WHOLLY DIGITAL

If General Motors had kept up with the technology like the computer industry has, we would all be driving $\$ 25.00$ cars that got 1,000 miles to the gallon. ${ }^{1}$

We are in an age of transformation in architecture, urbanism and the built environment. Artificial intelligence, machine learning, blockchain, virtual reality, Big Data, the internet of things and automated technologies such as industrial robotics and large-scale 3D printing are becoming more and more commonplace in architectural design, on construction sites, and in real estate developers and urban planning offices worldwide. The Fourth Industrial Revolution has led contemporaneity towards greater complexity, ever-increasing processing power and high degrees of interconnectedness. The way in which contemporary architectural practice has utilised digital technologies has, without a doubt, transformed the way in which the cultural production of the discipline is created and disseminated. Digital design tools and increased levels of computing power have given designers the ability to process huge amounts of information in order to test contextual, structural, environmental, formal and material variables, constraints and possibilities. More data about artefacts, objects, people, places and buildings can be collected and utilised in the design process than ever before, at a scale and detail unimaginable in previous decades. Building information modelling (BIM) softwares such as Revit have emerged as powerful programmes to enable this information to be shared, allowing for the relationship between architects, engineers and other subcontractors to be more effectively managed. Digital tools have entered into our contemporary consciousness as a discipline and their embedding into architectural design processes is evident throughout.

However, over twenty years on, the quote above by Microsoft CEO Bill Gates in 1997 where he emphasised transformations within the industrial manufacturing sector would be even more antithetical still if applied today to the construction industry. Electronic devices and cars have dramatically increased in quality and efficiency in terms of their use of digital manufacturing, and meanwhile in architecture buildings are being invaded by 'smart' gadgets and 'the Internet of Things' - technological developments which have very little to do with the processes of building, instead emphasising fully customisable inhabitation. Technologies such as robotic assembly and 3D printing - recent examples include SAM the robotic bricklayer by Construction Robotics and Winsun's 3D printed houses - are beginning to be used on construction sites, but the basic building blocks for buildings are typically planned and put together through processes that are still very much reliant on techniques developed in the 19th century with the advent of the Industrial Revolution. When one looks at examples such as those above it is easy to see that they merely replace common tasks of human labour. The promise and potential of digital design tools - mass customisation, infinite variation, quickness, cost effectiveness heralded by the 'digital designers' of the late 20th century such as Greg Lynn, Foreign Office Architects and Bernard Cache - has so far been not yet been translated into from the virtual to the physical world. As architect and theorist Neil Leach has aptly observed, there is "not yet a product as such that might be described as digital." Instead there exists a very slow, laborious, and highly discrete framework for the production of buildings. 
As a result, what are the social, economic and political consequences of a disruptive shift towards a wholly digital framework for the production of architecture? And in a world plagued by a housing crisis where millions live without adequate shelter across the world, how can this kind of production chain for architecture enable us to producing housing more quickly, more efficiently and with highly reduced costs that can respond to changes in family structures, in the way we organise our communities, and in how we relate to our physical and virtual environments? How can a wholly digital architecture of parts enable us to rethink the way in which we incorporate digital technologies and new social and economic frameworks into architectural design and construction practices that engage with wider communities that include architects and contractors, but also users/ inhabitants, policy-makers and/or other stakeholders? This paper therefore observes and provokes this contemporary condition, projecting a possible alternative method to design, fabricate and assemble architecture. To provide a typological context for the work, the ideas and projects presented here exclusively address the most banal and pervasive building typology worldwide: housing. The political and social frameworks surrounding housing both in relationship to architects, policy-making and construction provide ample ground for rethinking the role between design, technology and production in order to democratise, shorten and make more efficient the production chain.

The paper deploys the concept of the 'wholly digital', a term we have used in our work in the design studio Unit 19, part of Design Computation Lab at The Bartlett School of Architecture, UCL, for the last several years. The 'wholly digital' is aligned with the argument towards the discretisation of the spline that the architectural historian Mario Carpo argued for in his essay "Breaking the Curve" in Art Forum. ${ }^{3}$ As such it can also be contextualised within current work on the discrete by a younger generation of architects notably Gilles Retsin, Manuel Jimenez Garcia and Jose Sanchez. The paper aims to understand the data we design with as part of a framework of discrete, part-to-whole relationships across design and fabrication. This will be demonstrated not through a summative project description but through a series of themes using one project, semblr by Ivo Tedbury from 2017, to illustrate the main arguments.

\section{MOVING TOWARDS DISCRETE CONTINUITY}

As Mario Carpo has written the potential of Big Data - commonly understood in professional architectural practice as Building Information Modelling (BIM) - is its capacity to be a "tool or coping with, managing, and some would even say extolling complexity." ${ }^{\prime 4}$ However when one looks at the effect that the integration of BIM has had on professional practice, it has typically resulted in more and more specialists in order to manage and coordinate the large amounts of data. This then results in an increase in middle management to manage those who manage that information. With more and more labour required just to communicate the inputs of different specialisms involved in any given project there is an increased margin of error for miscommunication and failures in any given project. BIM's promise to streamline the communication required for architectural production is also dependent on, as Mark Klimt wrote back in 2011 in The Architect's Journal, "full and open collaboration in an industry that is still characterised by a blame culture and separateness between construction team members." 5

Therefore there is typically two distinct categories in the production of architecture: design and 'everything else' that happens afterwards. This is evermore clear when looking at one of the guidelines of professional practice in the United Kingdom: The Royal Institute of British Architects (RIBA) Plan of Work. ${ }^{6}$ This split is important to highlight as it is symptomatic of a discontinuity between the way digital tools and construction are linked. When it comes to building practices, advanced digital fabrication technologies such as industrial robots and $3 \mathrm{D}$ printers have so far been used most commonly as representative of human labour as mentioned above, or on the other end of the spectrum, as representational devices for architecture: in order to make copies, or replicas of objects. In addition, powerful and not-so-subtle political and legal structures maintain these conditions as the status quo in building design and construction - Klimt's blame culture being one of them. The legal system has not caught up with this evolution in the built environment professions where all parties are simultaneously an author and owner of a design.

It is hugely important to the evolution of building practices to better integrate and find continuity through a wholly digital architecture of parts between the virtual and physical, design and fabrication, architects and contractors. A wholly digital, discrete project for architecture is possible today. It is not, as Nick Scrnicek and Alex Williams wrote in Inventing the Future: Postcapitalism and a World Without Work "a free-floating project, since frameworks [...] already exist and have traction in the world." ${ }^{\prime \prime}$ It requires a recalibration of the ways in which they are already applied in order to critique, and evolve out of, the neoliberal mode of production within which we work today. It is a recognition that despite the adoption of BIM, Big Data, 3D printing, CNC milling industrial robots, virtual reality and artificial intelligence in architectural design, the way in which they have been used operates within that mode of production, succumbing to the constraints and protocols determined by its power structures. The power structures of neoliberalism privilege by and large those who already are in possession of wealth, i.e. capital. In the built environment this often equals land owners. The power of digital fabrication technologies therefore cannot transform the way in which work is completed as this would disrupt the way in which capital is accrued unless more radical steps are taken. 


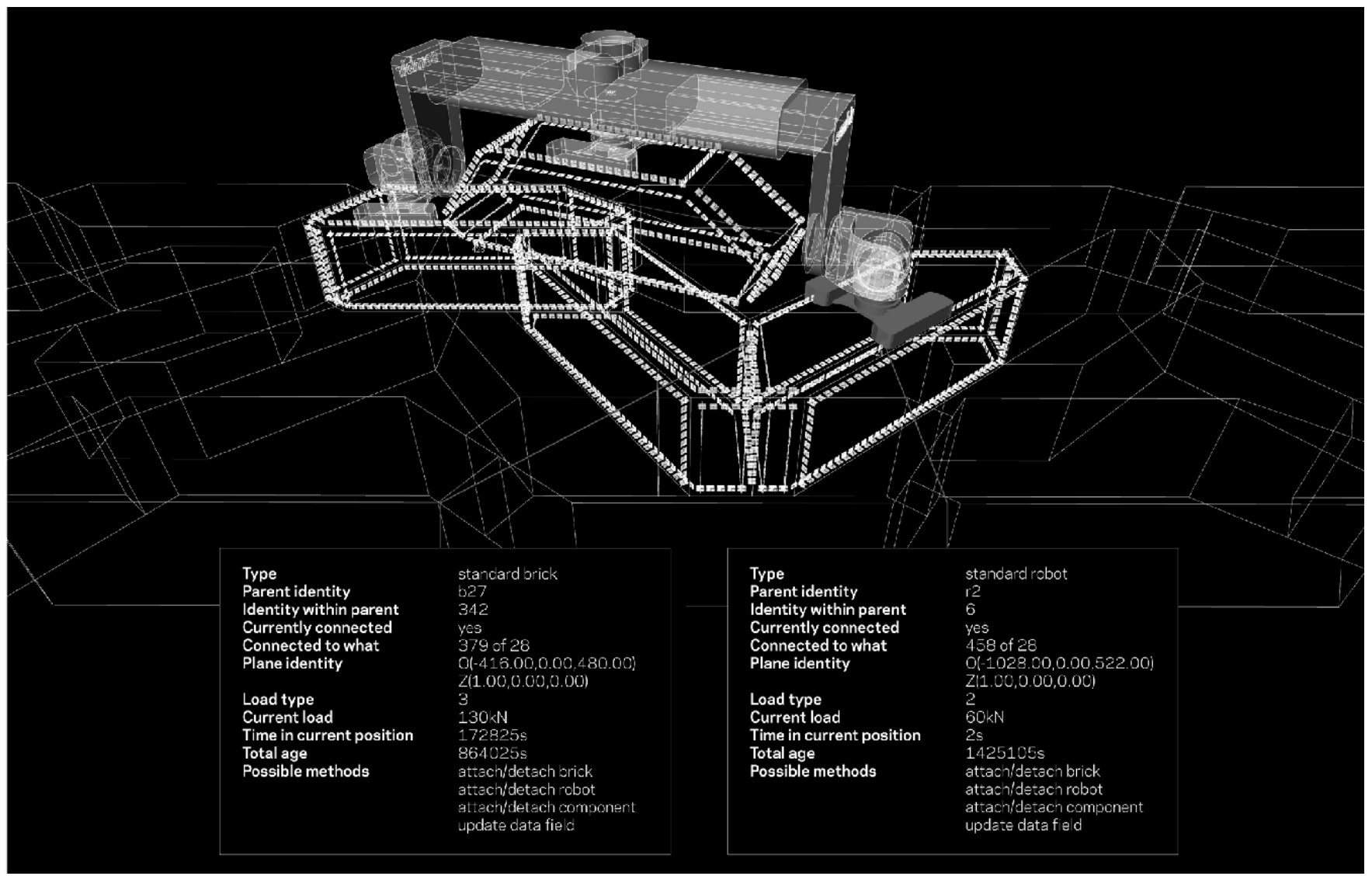

Figure 1: An exact virtual model of a physical robotic assembly process. Ivo Tedbury, Unit 19, Design Computation Lab, 2017.

As Scrnieck explains further in Platform Capitalism (2017), "in order to understand our contemporary situation, it is necessary to see how it links with what preceded it. Phenomena that appear to be radical novelties may, in historical light, reveal themselves to be simple continuities." 8 The use of digital technologies to further standardise architectural production, to produce more quickly and with more precision than the human labour that previously produced that work was capable of, is a convenient way to present these technologies as radical novelties. However they are simple continuities because of the very political and economic frameworks that sustain this particular culture. These technologies cannot be isolated tools to merely replace or manage the fallibilities of human labour. To therefore challenge the way that these technologies within architectural production are used in relationship to the politics and economics of the discipline of the built environment is to challenge the very nature of capitalism and neoliberalism itself. In effect, to be discrete, to move towards a wholly digital architecture of parts, is to enable continuity.

This approach of course has its roots in era of 20th century pre-fab architecture particularly in the architects Jean Prouvé (for example Maison Tropicale 1949-1952) and Buckminster Fuller (exemplified by his work Nine Chains to the Moon from 1938) who imagined entire production chains for their designs. However these architects were still limited by the normative and modernist paradigm for architectural syntax, i.e. column, beam, floor slab, stair, et cetera (although Fuller made some attempts at disrupting this with the Dymaxion House (first designed 1930, redesigned 1945). The Maison Dom-Ino (1914) by Le Corbusier is particularly exemplary of this paradigm. The elements that make up Maison Dom-Ino are constrained to their particular function: a column could only ever be a column, a beam could only ever be a beam. When we move away from building elements being specific to their architectural function and towards an architecture made of parts, or building blocks, then we begin move into the digital paradigm, which thinks of building blocks as open-ended, scalable, universal and versatile. Projects such as WikiHouse (2011-) attempt to take on board some of those qualities, however they are also restricted. It is digital in its means of fabrication (CNC milling) and in its use of open-source platforms. It is not digital in the sense that is is not scalable as it is too restricted to both the size of a sheet of plywood and the CNC bed. Scalability as we will see later is an important aspect of a digital architectural project. In addition WikiHouse provides mainly a structural frame for a house, it does not take into account servicing or required infrastructure, finishes or other aspects of what makes a house an inhabitable house. 


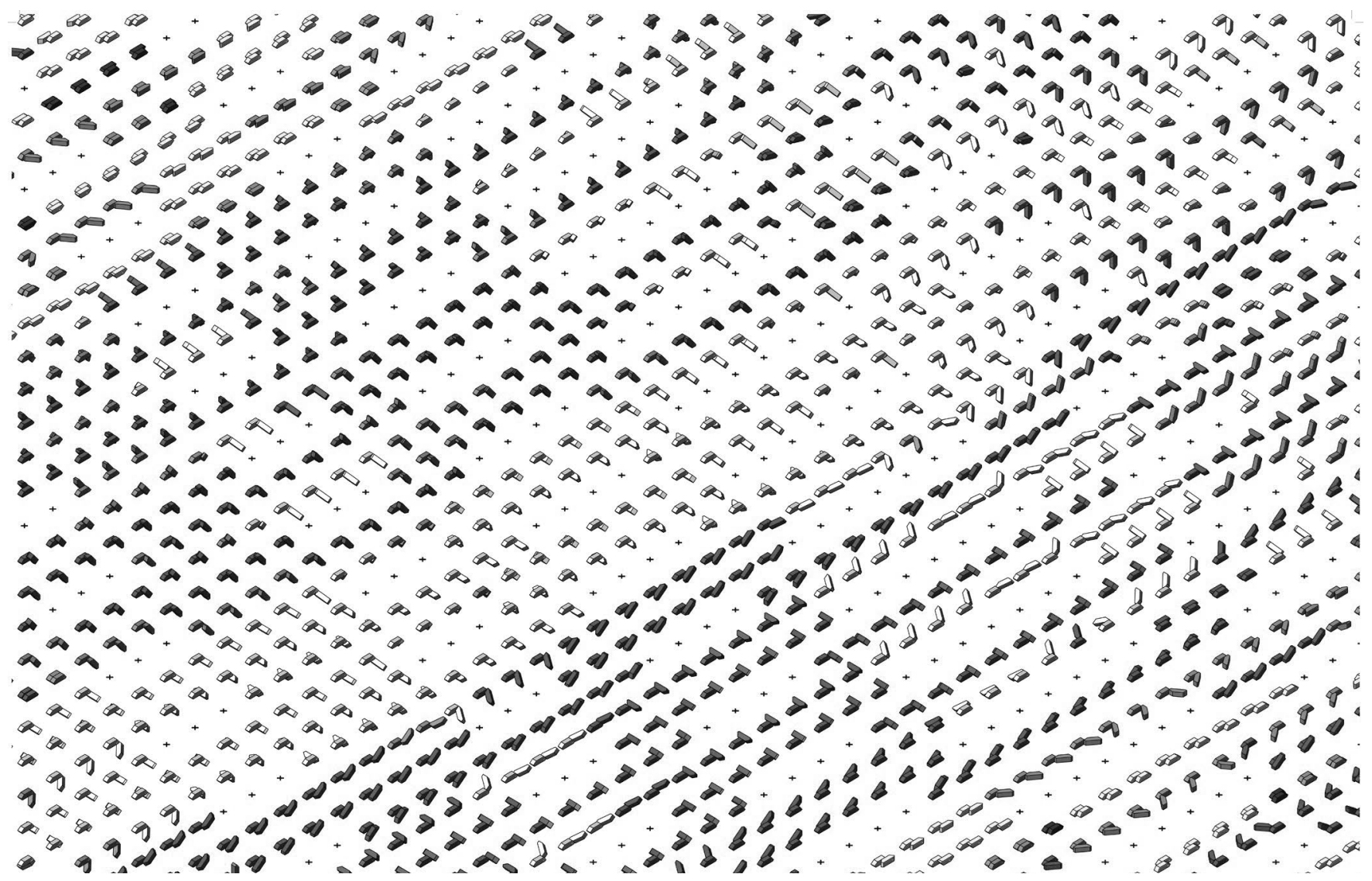

Figure 2: Generated combinations of all possible parts. Ivo Tedbury, Unit 19, Design Computation Lab, 2017.

\section{DIGITAL DISRUPTIONS}

Adopting a wholly digital approach forces rethinking of frameworks which already exist, that a provocation to our contemporary condition is possible. These frameworks include things such as automation, distribution networks, open-source technologies and means of user participation or interaction. The degree to which these are taken on board in the work is purposefully 'just' believable. In this the projects aim to shift today's Overton Window, the projects are plausible, not only in the far speculative future but in the near-now. Being wholly digital disrupts our traditional notions of part-to-whole relationships in architecture. If we begin to think of architecture as digital both in the design process and in the fabrication process (and not just in design or not just in the fabrication process), we can think of the parts that make up architecture as not overly-specific and closed building elements as in conventional building where every piece is designed and fabricated with high degrees of specificity and low tolerance. Instead, we can conceive of an architecture of parts as made of building blocks which can communicate across design to fabrication, as digital 'bits', and back again, part of a feedback loop rather than a linear system (figure 1). Building blocks can be distributed with an exactness to the virtual model, with high tolerances.
This requires a movement away from a master-architect model towards one where the architect design a process, or system of production. By designing a system of production, one can challenge the way in which automation is integrated into the production of architecture and not solely treat it as a means to an end. Each of the projects do not invent new technologies, but instead harness the power of existing technologies in a novel way. Automation is important, but automation does not mean exclusively robotic technologies. Automation in a wholly digital architectural project is engrained into all scales of how data is inputted into an overall framework - what is important is that building blocks can have enough agency to adapt automatically to changes in design constraints. It is also important to note that the design outcomes are only one outcome of many potential outcomes, but that each different possible outcome is syntactically exactly the same in each project. Project images again are presented merely as a means of visually explaining what this approach looks like as a project is developed within the paradigm of an architecture of parts. This further emphasizes the wholly digital nature of the project at large.

\section{DEPLOY, AUTOMATE, PARTICIPATE}

In order to move away from normative notions of architectural elements, the concept of building blocks is deployed. We refer 


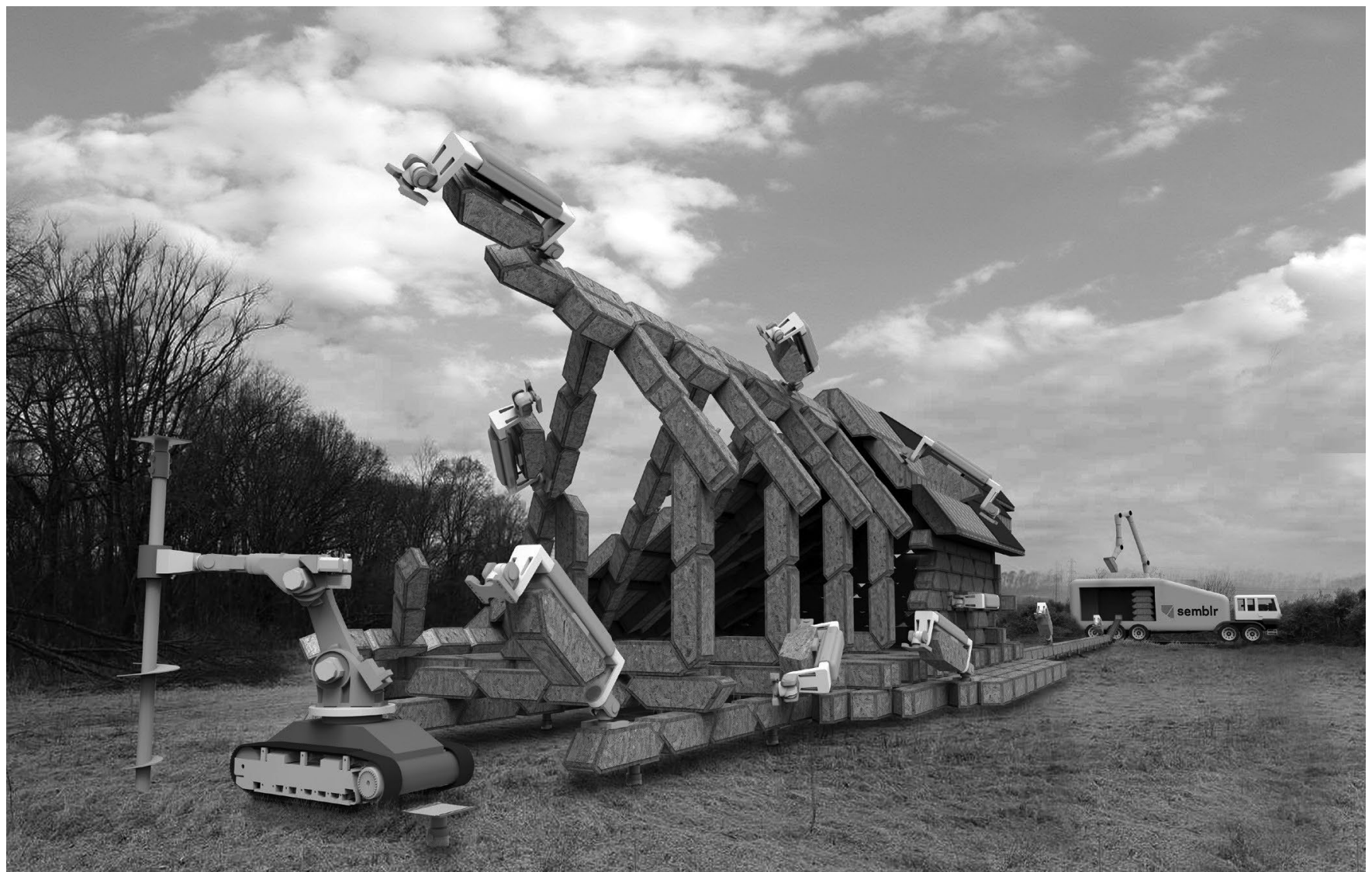

Figure 3: Autonomous semblr robots assembling housing structure. Ivo Tedbury, Unit 19, Design Computation Lab, 2017.

to these building blocks as 'voxels', or a three-dimensional pixel. The concept of the voxel is related to Neil Gershenfeld's concept of 'digital materials'. Gershenfeld, from the Centre for Bits and Atoms at Massachusetts Institute of Technology, recognised that while fabrication technologies are embedded with digital logics, materials were analogue. A digital material "assembled from a discrete set of parts, reversibly joined in a discrete set of relative positions and orientations." ${ }^{9}$ What digital materials enables is logic of connectivity between each discrete part in a 'kit of parts'. Each voxel therefore physically and virtually has a male-female connection which is the equivalent of the 0 and 1 in digital data, similar to Lego (figure 2).

This allows for an assembly and disassembly into new kits of parts continuously throughout the lifetime of the kit. In addition, the concept of digital materials enables the embedding of structural, spatial or material qualities - or any data - into each voxel or building block, and therefore into each kit of parts, or each different arrangement of the same kit of parts The best design outcomes - the way that parts are deployed, combine and aggregate - can be automated by the geometry (as well as material, production and other constraints or opportunities) and therefore, design agency, of the part itself. Furthermore, parts are scalable, able to 'turn on' or 'turn off' more data as scales shift from micro to macro.
When working with building blocks as digital materials, it is important to note that there is a process of instruction on the part of the designer, i.e. what is the particular thing one wants the building block that makes up the kit of parts to do? As a result, particular qualities - whether they are spatial, structural or environmental - can get emphasized or privileged depending on the way the kit of parts of the building blocks is assembled in a discrete system for architecture. This results in the emergence of patterns and possibilities, both architecturally (spatially) and syntactically (structurally). The design approach is simultaneously both 'top-down' and 'bottom-up', enabling for authorship or ownership of the design process (as an intuitive and creative act) and for discovery in how a particular set of rules may behave or give different outcome when given certain constraints within the design process (i.e. as a logical and rational set of operations).

It is through evaluation of all different outcomes through architectural constraints that those outcomes are either dismissed as irrelevant or not appropriate to the design or taken onboard as options to develop the kit of parts further. Typically, a kit of parts privileges one particular set of design constraints, i.e. structural, material or spatial. By utilizing this approach, a single part can hold enough information or data to be able to design, fabricate and assemble parts for an entire building. This streamlines the relationship between process and outcome (figure 3). 
What a wholly digital model for architecture also enables is the role of the user to participate through inputting into the assembly of a particular spatial configuration their own set of desired outcomes. The user in this kind of wholly digital architectural project can be defined in several ways and at several scales, i.e. at the micro scale or towards the macro scale. The micro scale operates at the scale of the human body, or the inhabitant, while the macro scale is more at the scale of the system, i.e. in terms of the production chain for the fabrication or assembly of the building and how it relates to political, economic or material constraints. The micro scale could be said to be about particular behavior or action of a person, while the macro is about how that behavior operates within a framework. Each scale holds huge amounts of potential for the engagement of the user enabling surprising and novel outcomes. The architect sets a framework or system for the user/inhabitant to customize.

What is interesting about the differences between scales is the way in which user participation could be communicated. At the scale of the inhabitant, s/he may have access to a set of building blocks or kit of parts that $s$ /he can use to customize her living space, communicating innate wants or desires through her individual ability to act on or within their space in an architectural way. The behavior is generally localized and not particularly scalable - in effect this is merely playing out the "folk politics" of Srnicek and Williams in regard to user participation in political actions such as the Occupy Movement. ${ }^{11}$ The macro scale however involves the compete revision of the production chain to 'cut out the middle man' and not use technology as a replacement for human labor, instead empowering users to collaborate and pool resources in order to procure capital and revise existing procurement frameworks.

The potential of a wholly digital architecture therefore must be to move beyond the paradigm of mass-customization from the late 20th century. This can be done by envisioning production chains for housing as bringing together large user groups, compiling and democratizing access to data and tools and introducing users into the processes of design and fabrication. It is important that users become vital to this process, feeding back into the system their own knowledge in order to better inform and predict design outcomes.

\section{DEPLOY, AUTOMATE, PARTICIPATE}

The projects that were presented in this paper are attempts to propose ways to disrupt the current way that we produce architecture. To make these projects a reality in the near-now requires a cultural shift or disruption in the discipline. This shift needs to be towards more distributed, rather than centralized, forms of capital. It also requires an overhaul of the way in which projects are procured, designed, produced and constructed. The projects included here merely illustrate the possibility of just how close we could be to discrete model for architectural production which provides continuity between design and construction. Automated technologies such as industrial robots but also including CNC-milling, vacuumforming and 3D printing should not just be a replacement for simple, repetitive tasks of human labor. They provide an opportunity to rethink the role of the architect, client, user or inhabitant and contractor. The political and social frameworks surrounding housing both in relationship to architects, policy-making and construction provide ample ground for rethinking the role between design, technology and production in order to democratize, shorten and make more efficient the production chain. This shift in paradigms towards a wholly digital architecture of parts is where the future lies today in architectural design.

\section{ENDNOTES}

1. Bill Gates, speaking at COMDEX, 1997.

2. Neil Leach, "There is No Such Thing as Digital Design," in David Gerber, Mariana Ibanez (eds.), Paradigms in Computing: Making, Machines, and Models for Design Agency in Architecture, Los Angeles: eVolo Press, 2014, 148-158.

3. Mario Carpo, 'Breaking the Curive: Big Data and Design," Art Forum, Vol. 52, No. 6, February 2014.

4. Ibid.

5. Mark Klimt, "Legalese: the problem with BIM." The Architect's Journal, EMAP Publishing Ltd, 2011. https://www.architectsjournal.co.uk/opinion/legalesethe-problem-with-bim/8617331.article, accessed 12 May 2018.

6. "RIBA Plan of Work." Royal Institute of British Architects, 2017, https://www. architecture.com/knowledge-and-resources/resources-landing-page/ribaplan-of-work, accessed 05 May 2018.

7. Nick Srnicek and Alex Williams, Inventing the Future: Postcapitalism and a World Without Work, Verso, 2015, 107.

8. Nick Srnicek, Platform Capitalism, Polity Press, 2016, 9.

9. Neil Gershenfeld, Matthew Carney, Benjamin Jenett, Sam Calisch, and Spencer Wilson, "Macrofabrication with Digital Materials: Robotic Assembly," $A D$ : Special Issue: Material Synthesis: Fusing the Physical and Computational, Vol. 85 (5). Wiley, 2015, 123. 